\title{
PERIODIC CYCLES AND BIFURCATION CURVES FOR ONE-DIMENSIONAL MAPS WITH TWO DISCONTINUITIES
}

\author{
GIAN-ITALO BISCHI ${ }^{1}$, LAURA GARDINI ${ }^{2}$, UGO MERLONE $^{3}$ \\ 1,2DIPARTIMENTO DI ECONOMIA E METODI QUANTITATIVI UNIVERSITÀ DI \\ URBINO VIA SAFFI N. 42, I-61029 URBINO (ITALY) \\ E-MAIL: BISCHI@UNIURB.IT \\ E-MAIL: GARDINI@UNIURB.IT \\ ${ }^{3}$ DIPARTIMENTO DI STATISTICA E MATEMATICA APPLICATA, UNIVERSITÀ DI \\ TORINO CORSO UNIONE SOVIETICA 218/BIS I-10134 TORINO, ITALY \\ E-MAIL: MERLONE@ECON.UNITO.IT
}

\begin{abstract}
Starting from a family of discontinuous piece-wise linear one-dimensional maps, recently introduced as a dynamic model in social sciences, we propose a geometric method for finding the analytic expression of the bifurcation curves, in the space of the parameters, that bound the regions characterized by the existence of stable periodic cycles of any period. The conditions for the creation and the destruction of periodic cycles, as well as the analytic expressions of the bifurcation conditions, are obtained by studying the occurrence of border-collision bifurcations. In this paper we consider the case of maps formed by three linear portions separated by two discontinuity points. After summarizing the bifurcation structure associated with one-dimensional maps with only one discontinuity point, we show how this is modified by the introduction of a second discontinuity point. Finally we show how the considered map can be obtained as the limit case of a family of continuous maps as a parameter is increased without bounds, and we show how the low period cycles, which are typical of the discontinuous map we consider, emerge from the more complex (i.e. chaotic) behaviors observed in the continuous maps when a parameter value is large enough. From the point of view of the social application the increasing values of the parameter can be interpreted as higher degrees of impulsivity of the agents involved in binary decisions.
\end{abstract}

\footnotetext{
JOURNAL OF DYNAMICAL SYSTEMS \& GEOMETRIC THEORIES

VOL. 7, NUMBER 2 (2009) 101-123.

(C)TARU PUBLICATIONS
} 
AMS Classification: 37G35, 34C25, 37E05, 37E15, 37N40

Keywords: Discrete dynamical systems, Piecewise linear map, Border collision bifurcation, Periodicity tongues.

\section{INTRODUCTION}

Since the Seventies of the last century, plenty of papers and books devoted to dynamic and geometric properties of nonlinear discrete dynamical systems have been published, giving rise to a flourishing literature on the qualitative study of the asymptotic behaviors and bifurcation theory for discrete dynamical systems, represented by the iteration of differentiable one-dimensional maps (see e.g. [8], [11], [19], [49], [39]). However, in the recent literature on dynamic modelling applied to the description of engineering, economic and social systems, several models are ultimately expressed in the form of iterated piecewise differentiable functions, which may either be continuous (see e.g. [9], [10], [22], [23], [24], [16], [35], [36], [45], [42], [43], [17]) or discontinuous, with one or more discontinuity points ([38], [37], [44], [46], [47], [7], [48]). The bifurcations involved in such class of maps are often described in terms of the so called border-collision bifurcations. We can classify as border-collision any qualitative change in the properties of a dynamical system due to a contact between an invariant set and a boundary (or border) of its region of differentiability. The term border-collision bifurcation was used for the first time by Nusse and Yorke in [33] (see also in [34]) and it is now widely used in this context, i.e. for piecewise smooth maps, although the study and description of such bifurcations started several years before these papers. For example, Leonov ([26], [27]) described several bifurcations of that kind, and gave a recursive relation to find the analytic expression of the sequence of bifurcations occurring in a one-dimensional piecewise linear map with one discontinuity point, which is still almost unknown, except for a limited number of researchers among which Mira ([31], [32]), Maistrenko et al. [28], [29], [30]. See also the results obtained by Feigen in 1978, re-proposed in di Bernardo et al. [12], [13]. These authors already described the contact bifurcations now called of border collision, but using different names and notations.

These kinds of bifurcations are now widely studied and gave rise to a flourishing literature in the last years, mainly because of their relevant applications in Electrical 
PERIODIC CYCLES AND BIFURCATION .. — JDSGT VOL. 7, NUMBER 2 (2009) 103

and Mechanical Engineering. In fact, several papers on piecewise smooth dynamical systems and border collision bifurcations have been motivated by the study of models used to describe particular electrical circuits or systems for the transmission of signals ([28], [29], [30], [12], [13], [3], [4], [5], [25], [14], [15], [20], [50], [51], [52], $[1],[2])$.

In this paper we analyze a family of discontinuous piecewise linear maps proposed by Bischi and Merlone [6] to model the dynamic evolution of binary decisions in a population of "impulsive individuals" under the influence of social externalities. In fact, Bischi and Merlone ([6]) presented the following discrete-time adaptive dynamic model, based on the qualitative properties described by Schelling ([40], [41]), to represent an adjustment process of repeated binary choices under social externalities:

(1)

$$
x_{t+1}=f\left(x_{t}\right)= \begin{cases}x_{t}+\delta_{A} g\left[\lambda\left(A\left(x_{t}\right)-B\left(x_{t}\right)\right)\right]\left(1-x_{t}\right) & \text { if } A\left(x_{t}\right) \geq B\left(x_{t}\right) \\ x_{t}-\delta_{B} g\left[\lambda\left(B\left(x_{t}\right)-A\left(x_{t}\right)\right)\right] x_{t} & \text { if } A\left(x_{t}\right)<B\left(x_{t}\right)\end{cases}
$$

where $x \in[0,1]$ represents the fraction of players that choose the option $A$, characterized by a payoffs function $A(x)$, whereas the complementary fraction $(1-x)$ chooses the opposite strategy $B$ that has payoff $B(x), \delta_{A}, \delta_{B} \in[0,1]$ represent switching propensities and $g: \mathbb{R}_{+} \rightarrow[0,1]$ is a continuos and increasing function such that $g(0)=0$ and $\lim _{z \rightarrow \infty} g(z)=1$. The positive parameter $\lambda$ representing the speed of reaction of agents - small values of $\lambda$ imply more inertia, while, on the contrary, larger values of $\lambda$ imply more reactive agents. In [7] the limiting case when $\lambda$ tends to infinity has been considered, which represents agents that immediately switch their strategies even when the difference between payoffs is extremely small, also called impulsive agents. Under this assumption the function $f$ becomes discontinuous:

$$
x_{t+1}=f_{\infty}\left(x_{t}\right)=\left\{\begin{array}{lll}
\left(1-\delta_{A}\right) x_{t}+\delta_{A} & \text { if } & B\left(x_{t}\right)<A\left(x_{t}\right) \\
x_{t} & \text { if } & B\left(x_{t}\right)=A\left(x_{t}\right) \\
\left(1-\delta_{B}\right) x_{t} & \text { if } & B\left(x_{t}\right)>A\left(x_{t}\right)
\end{array}\right.
$$

In [7] this limiting case has only been considered with monotonic payoff functions that intersect in one interior equilibrium point, and for the corresponding piecewise 
linear map with one discontinuity point conditions for the creation and the destruction of periodic cycles, as well as the analytic expressions of the bifurcation curves, have been derived. In this paper the same limiting case of impulsive agents is considered under the realistic assumption of non monotonic payoff curves (see Schelling, [40], page 414, Granovetter, [18], page 1438) that describe situations where both a low diffusion as well as an excessive diffusion of a given collective choice induces an extreme form of over-reaction leading to fast transition to the opposite choice, i.e. we assume that $A(x)>B(x)$ for $d_{1}<x<d_{2}$ and $A(x)<B(x)$ otherwise. So, in this paper we are interested in extending the results given in [7] to the case with two discontinuities, by using methodologies similar to those used in [48] for a different model with two discontinuities.

The structure of the paper is the following. In section 2, starting from the one discontinuity map, we introduce the map with two discontinuities and provide a formal analysis of the bifurcation curves; in section 3 we show how the map we analyze can be obtained as the limiting case of a continuous map. Finally, the last section is devoted to some concluding remarks.

\section{A FAMily of PIECEWISE LINEAR MAPS With TWO DisCONTINUities}

The family of iterated maps $T:[0,1] \rightarrow[0,1]$ considered in the present paper has the form:

$$
x^{\prime}=T(x)= \begin{cases}\left(1-\delta_{B}\right) x & \text { if } \quad x<d_{1} \text { or } x>d_{2} \\ x & \text { if } \quad x=d \\ \left(1-\delta_{A}\right) x+\delta_{A} & \text { if } \quad d_{1}<x<d_{2}\end{cases}
$$

with parameters subject to the following constraints $([6])$ :

$$
\begin{gathered}
0<d_{1}<d_{2}<1 \\
0<\delta_{A}<1, \quad 0<\delta_{B}<1
\end{gathered}
$$

Defining $m_{1}=1-\delta_{B}$ and $m_{2}=1-\delta_{A}$ the map can be rewritten as:

$$
x^{\prime}=T(x)= \begin{cases}T_{R}(x)=m_{1} x & \text { if } \quad x<d_{1} \text { or } x>d_{2} \\ x & \text { if } \quad x=d \\ T_{L}(x)=m_{2} x+1-m_{2} & \text { if } \quad d_{1}<x<d_{2}\end{cases}
$$


The cases with one discontinuity point occur if either $d_{1}=0$ or $d_{2}=1$, as in these cases the map (3) becomes

$$
x^{\prime}=M_{1}(x)=\left\{\begin{array}{lll}
\left(1-\delta_{A}\right) x+\delta_{A} & \text { if } & x<d_{2} \\
x & \text { if } & x=d_{2} \\
\left(1-\delta_{B}\right) x & \text { if } & x>d_{2}
\end{array}\right.
$$

or

$$
x^{\prime}=M_{2}(x)=\left\{\begin{array}{lll}
\left(1-\delta_{B}\right) x & \text { if } & x<d_{1} \\
x & \text { if } & x=d_{1} \\
\left(1-\delta_{A}\right) x+\delta_{A} & \text { if } & x>d_{1}
\end{array}\right.
$$

respectively. These two maps, characterized by a decreasing jump and an increasing jump, respectively, have been recently studied in [7] where an analysis of the socalled "principal tongues" ([28], [29], [30], [12], [13], [4], [1], [2]) or "tongues of first degree" (Leonov, [26], [27], Mira [31], [32]) is provided. We recall that the principal tongues are regions, in the parameters' space, where a periodic cycle of period $k$ exists, with one periodic point on a side of the discontinuity point and the remaining $(k-1)$ points on the other side (for any integer $k>1$ ).

For the case with two discontinuities, considered in this paper, let us start from the description of the possible attractors of the map. Two examples are shown in Fig. 1, where two different cases of coexisting attractors are shown, respectively given by the fixed point $x=0$ and a stable cycle of period 3 (Fig. 1a) or the same fixed point with a stable cycle of period 2 (Fig. 1b). It is worth noticing that in this model the origin is always an attractor: It may be the only one or it may coexist with an attracting cycle of period $k>1$, according to the values of the four parameters $d_{1}, d_{2}, \delta_{A}$ and $\delta_{B}$. In the following we shall prove the existence of stable periodic cycles in analytically defined regions of the space of the parameters, as well as the analytic conditions for their creation/destruction through border collision bifurcations.

In the case of coexisting attractors, it is also important to bound the sets of initial conditions that generate trajectories that converge to one or to the other, i.e. the respective basins of attraction. Indeed, we only have the two following possibilities: 

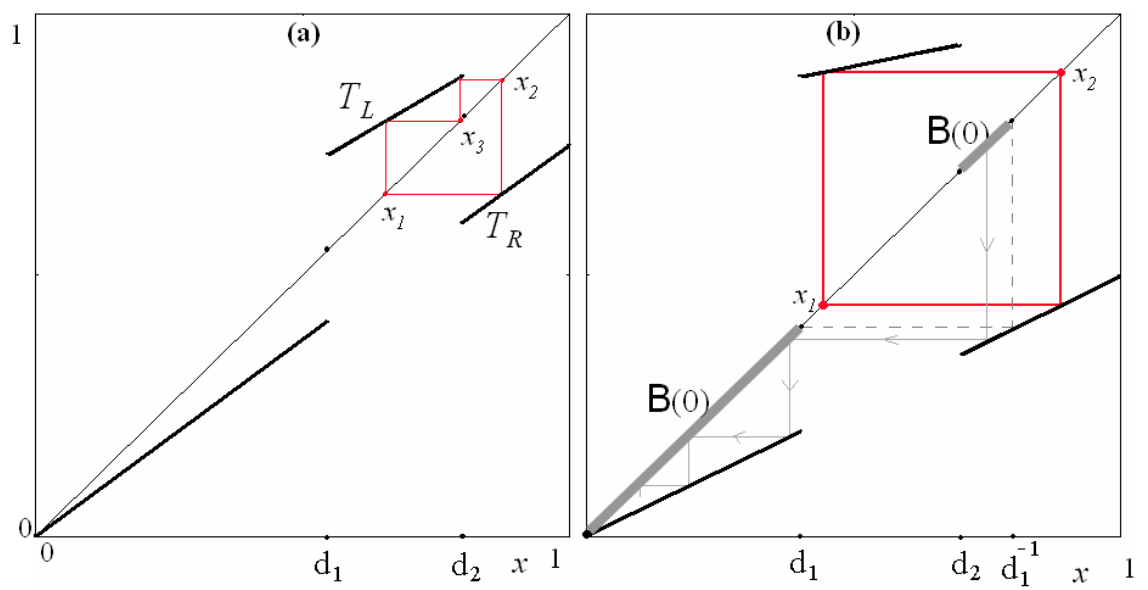

Figure 1. Map (4), coexistence of two attractors. (a) With parameters $m_{1}=0.75, m_{2}=0.6, d_{1}=0.55, d_{2}=0.8$, the two attractors are the origin and a cycle of period 3. (b) With parameters $m_{1}=0.5, m_{2}=0.2, d_{1}=0.4, d_{2}=0.7$ the two attractors are the origin and a cycle of period 2 .

(a) each of the two basins is made up of only one interval, separated by the discontinuity point $d_{1}$ (as in the case shown in Fig. 1a), so we have that the basin of the origin is the first interval: $B(O)=\left[0, d_{1}\right.$ [ while the other points converge to the attracting cycle: $\left.B(C)=] d_{1}, 1\right]$;

(b) each of the two basins is made up of two or more intervals (an example is shown in Fig. 1b), separated by the two discontinuity points $d_{1}$ and $d_{2}$ and their preimages. The immediate basin of the origin is clearly the segment $\left[0, d_{1}[\right.$ so that the whole basin is given by this segment and all its preimages: $B(0)=$ $\cup_{j \geq 0} T^{-j}\left(\left[0, d_{1}[)\right.\right.$, while the complementary region in $[0,1]$ gives the basin of the cycle: $B(C)=[0,1] \backslash B(0)$.

We notice that for the example in Fig. 1b the basin of the origin $B(O)$ is made up of the two intervals: $B(O)=\left[0, d_{1}[\cup] d_{2}, d_{1}^{-1}\right.$ [ where $d_{1}^{-1}=T_{R}^{-1}\left(d_{1}\right)=d_{1} / m_{1}$, and the complementary region gives the basin of the cycle: $\left.B(C)=] d_{1}, d_{2}[\cup] d_{1}^{-1}, 1\right]$.

From the above description it is plain that the qualitative change in the structure of the basins depends on the position of the rank-1 preimage of $d_{1}$, denoted by $d_{1}^{-1}$ : if it does not exist we have the situation (a), as shown in Fig. 1a; if it exists, we get the 
PERIODIC CYCLES AND BIFURCATION .. — JDSGT VOL. 7, NUMBER 2 (2009) 107

situation (b), hence the global dynamics are quite different. The bifurcation leading from situation (a) to (b) (or vice-versa) occurs when $d_{1}^{-1}$ appears (disappears) through a so called "border collision bifurcation", occurring when $d_{1}^{-1}=T_{R}^{-1}\left(d_{1}\right)$ has a contact with $d_{2}$, i.e. when $d_{1} / m_{1}=d_{2}$, or, equivalently, when $m_{1}=d_{1} / d_{2}$, i.e. $\delta_{B}=\left(d_{2}-d_{1}\right) / d_{2}$.

If we define

$$
\delta_{B}^{*}=\frac{d_{2}-d_{1}}{d_{2}}
$$

then the following proposition easily follows from the arguments given above:

Proposition 1. (a) When $\delta_{B}<\delta_{B}^{*}$ then there are two coexistent attractors, the origin and $a k$-cycle $C_{k}$ for some integer $k$, and each basin of attraction is formed by one interval: $\mathrm{B}(O)=\left[0, d_{1}[\right.$ and $\left.B(C)=] d_{1}, 1\right]$.

(b) When $\delta_{B}>\delta_{B}^{*}$ then either the origin is the only attractor with basin $B(O)=$ $[0,1]$ or there are two coexisting attractors, the origin and a $k$-cycle $C_{k}$ for some integer $k$. The basins of the origin is given by $B(0)=\cup_{j \geq 0} T^{-j}\left(\left[0, d_{1}[)\right.\right.$, while the complementary region in $[0,1]$ gives the basin of the $k$-cycle: $B\left(C_{k}\right)=[0,1] \backslash B(0)$.

According to this Proposition, when the origin is not the only attractor, it coexists with a stable cycle of period $k$. Indeed, we shall see that stable cycles $C_{k}$ of any period $k>1$ can exist, and we shall also distinguish several qualitatively different cycles among those that have the same period $k$. In fact, the existence of a $k$-cycle is associated only with a portion of the map, and precisely with the two parts of the map located on the right of the first discontinuity point. For example, in the range of parameters considered in part (a) of Proposition 1, we have two qualitatively different dynamics separated by the discontinuity point $d_{1}$, and in the interval $\left.] d_{1}, 1\right]$ our model is qualitatively the same of "a discontinuous map with only one jump of decreasing type", whose dynamic behaviors are similar to those studied in [7]. So, for initial conditions taken in the interval ] $\left.d_{1}, 1\right]$ we already know that all the possible cycles $C_{k}, k>1$, may exist, with periodic points in the intervals $\left.] d_{1}, d_{2}[\cup] d_{2}, 1\right]$. Instead, in the parameter range considered in part (b) of Proposition 1 we may have either coexisting cycles or not. In the first case, i.e., when two different attractors coexist, then the periodic points of the $k$-cycle are all included in $\left.] d_{1}, d_{2}[\cup] d_{2}, 1\right]$. An example of the different periods which may exist is shown in the two bifurcations diagrams of Fig. 2 . 

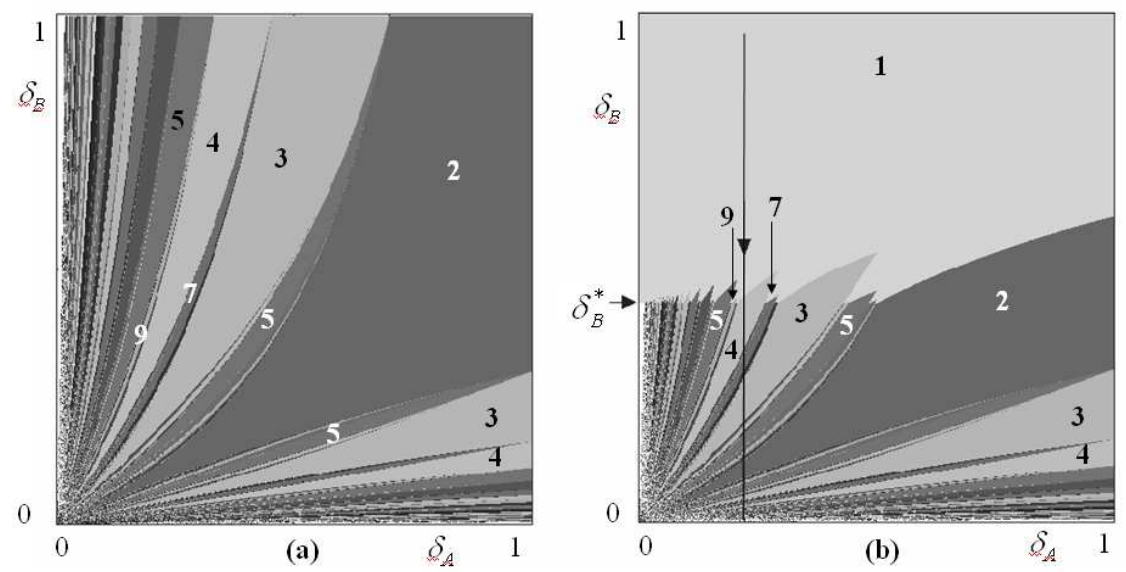

Figure 2. Two-dimensional bifurcation diagrams in the plane $\left(\delta_{A}, \delta_{B}\right)$ of the map $T$. In (a) $d_{1}=0.0$ and $d_{2}=0.7$. In (b) $d_{1}=0.4$ and $d_{2}=0.7$.

Fig. 2a shows the bifurcation diagram in the case $d_{1}=0$ and $d_{2}=0.7$, i.e. when the map has only one discontinuity point with decreasing jump. In this case stable cycles $C_{k}$ exist for any $k>1$, which involve the two branches of the map around $d_{2}$, say the left branch $T_{L}(x)$, for $0<x<d_{2}$, and the right branch $T_{R}(x)$, for $d_{2}<x<1$. By contrast, in Fig. 2b we show the dynamic effect associated with the introduction of the other discontinuity point, $d_{1}$, i.e. another branch which includes the stable fixed point at the origin. In Fig. $2 \mathrm{~b}$ it can be seen that for $0<\delta_{B}<\delta_{B}^{*}$ the two bifurcation diagrams are identical, and changes only occur for $\delta_{B}>\delta_{B}^{*}$. The meaning of the gray tonalities is that when the parameters are taken in the white region the origin is the only attractor, while in the grey-shaded regions the origin coexists with a $k$-cycle, whose period $k$ is different in regions with different grey tones. In Fig. 2 only a few periodicity tongues are visible, but we shall demonstrate that infinitely many exist, as periodicity tongues of all periods $k>1$ exist, bounded by bifurcation curves corresponding to the occurrence of border-collision bifurcations. We shall also give the analytic expression of the bifurcation curves that bound the principal tongues.

In Fig. 3 we show a one-dimensional bifurcation diagram showing the state variable $x$ as the parameter $\delta_{B}$ is decreased along the line shown in Fig. 2b, i.e. at 


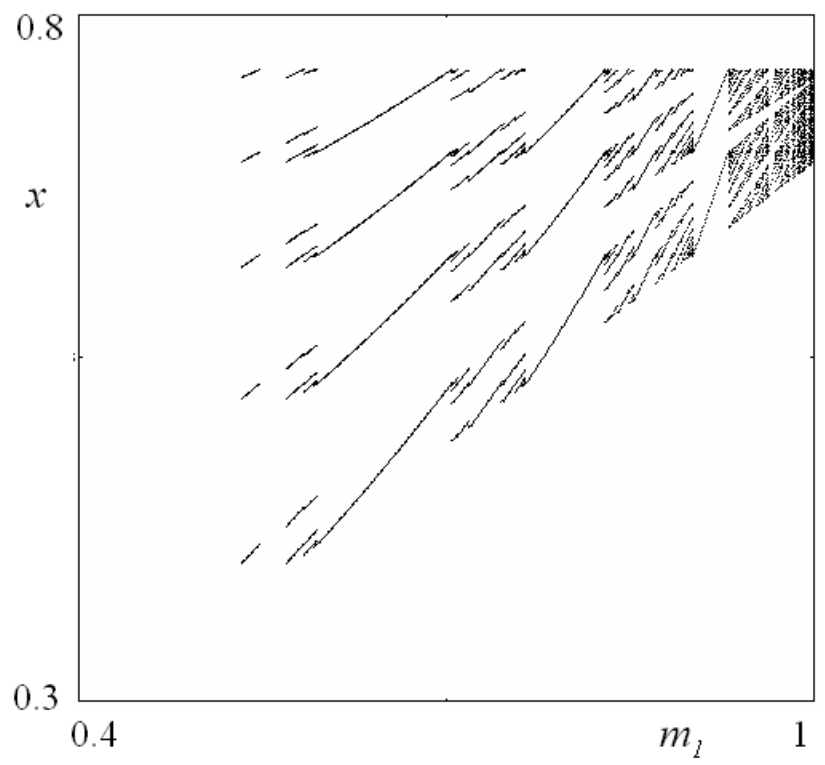

Figure 3. One-dimensional bifurcation diagram of the state variable $x$ as the parameter $\delta_{B}$ is decreased along the line shown in Fig.2b, with a fixed value $\delta_{A}=0.2$.

the fixed value of $\delta_{A}=0.2$. Even in this picture, only a few $k$-cycles are detectable, however if the scale is enlarged, i.e. after a zoom-out of any portion of the bifurcation diagram, more and more different cycles appear between any pair of the main cycles visible in Fig. 3.

Before entering the detail of the border collision bifurcations that cause the creation and the destruction of the cycles, we want to stress that any cycle, when it exists, is asymptotically stable. In fact, the stability of a $k$-cycle is given by the slope (or eigenvalue) of the function $T^{k}=T \circ \ldots \circ T$ ( $k$ times) in a periodic point of the cycle, which is also a fixed point of the map $T^{k}$. Then, by the chain rule, the eigenvalue in any one of the periodic points is given by the product of the $k$ slopes of the map in the $k$ periodic points, and assuming that the cycle has $p$ points on the left side and $(k-p)$ on the right side, the eigenvalue is given by $m_{1}^{p} m_{2}^{(k-p)}$ which, in our assumptions, is always positive and less than 1.

We also stress that a periodic cycle can only exist with periodic points belonging to the two linear pieces in the range $\left[d_{1}, 1\right]$, that is, they are associated with $T_{L}(x)$, 
in the range $d_{1}<x<d_{2}$, which we shall call left side $L$ henceforth, and of the map $T_{R}(x)$, in the range $d_{2}<x<1$, which we shall call right side $R$. As we shall see, a $k$-cycle with $k>1$ may have $p$ points on the left side $L$ and $(k-p)$ on the right side $R$, for any $k>1$ and $p<k$. In particular, a "main" or "principal" $k$-cycle is a cycle having period $k$ with one point on one side and $(k-1)$ points on the other side, whose periodicity regions are also called as "tongues of first degree of complexity" (Leonov, [26], [27], Mira [31], [32]).

Let us start with the determination of the border-collision bifurcation curves associated with the existence of a "principal $k$-cycle", with one point on the left side, $L$, and $(k-1)$ points on the right side, $R$. The condition that starts the existence is that the discontinuity point $x=d_{2}$ is a periodic point to which we apply, in the sequence, the maps $T_{L}, T_{R}, \ldots, T_{R}$. For example, the starting condition related with the 3 -cycle $(k=3)$, is $T_{R} \circ T_{R} \circ T_{L}\left(d_{2}\right)=d_{2}$. Then the $k$-cycle with periodic points $x_{1}^{*}, \ldots, x_{k}^{*}$, numbered with the first point on the left side (i.e. $x_{1}^{*}<d_{2}$, $x_{i}^{*}>d_{2}$ for $\left.i=2, \ldots, k\right)$ satisfies $x_{2}^{*}=T_{L}\left(x_{1}^{*}\right), x_{3}^{*}=T_{R}\left(x_{2}^{*}\right), \ldots, x_{1}^{*}=T_{R}\left(x_{k}^{*}\right)$. The border collision which starts the periodicity region occurs when $x_{1}^{*}=d_{2}$ and this cycle, in standard conditions, ends to exist when the last point, $x_{k}^{*}$, merges with the discontinuity point, that is, $x_{k}^{*}=d_{2}$, which may be stated as the point $x=d_{2}$ is a periodic point to which we apply, in the sequence, the maps $T_{R}, T_{L}, T_{R}, \ldots, T_{R}$.

The standard conditions refers to the portion of the bifurcation diagram in which we are sure that the discontinuity point $d_{1}$ is not influencing the analysis, that is, this certainly occurs for $\delta_{B}<\delta_{B}^{*}$. But when we have $\delta_{B}>\delta_{B}^{*}$ then the "starting condition" no longer occurs, which means that a periodicity tongue cannot be created any longer, and those already created (for $\delta_{B}<\delta_{B}^{*}$ ) have a closure which behaves differently: instead of the standard closing condition $\left(x_{k}^{*}=d_{2}\right)$ the cycle ends to exist when the last point, $x_{k}^{*}$, merges with the boundary of the basin of the origin, that is, when $x_{k}^{*}=d_{1}^{-1}$, that is, when $x_{k}^{*}=d_{1} / m_{1}$. The formalization of the arguments given above allows us to get the analytic expression of the border collision bifurcation curves. In fact, let us consider the discontinuity point $d_{2}$ and 
apply the maps $T_{L}, T_{R}, \ldots, T_{R}$ :

$$
\begin{aligned}
x_{1} & =d_{2} \\
x_{2} & =T_{L}\left(x_{1}\right)=m_{2} d_{2}+1-m_{2} \\
x_{3} & =T_{R}\left(x_{2}\right)=m_{1}\left(m_{2} d_{2}+1-m_{2}\right) \\
x_{4} & =T_{R}\left(x_{3}\right)=m_{1}^{2}\left(m_{2} d_{2}+1-m_{2}\right) \\
& \ldots \\
x_{k+1} & =T_{R}\left(x_{k}\right)=m_{1}^{(k-1)}\left(m_{2} d_{2}+1-m_{2}\right)
\end{aligned}
$$

and a $k$-cycle occurs when $x_{k+1}=x_{1}$. i.e.

$$
d_{2}=m_{1}^{(k-1)}\left(m_{2} d_{2}+1-m_{2}\right)
$$

from which we obtain the analytic expression of the starting bifurcation curve:

$$
m_{2}=\frac{d_{2}-m_{1}^{(k-1)}}{m_{1}^{(k-1)}\left(d_{2}-1\right)}
$$

of a cycle with one point on the left side, $L$, and $(k-1)$ points on the right side, $R$, which holds for $\delta_{B}<\delta_{B}^{*}$. From the above expression we can also explicitly write the periodic points as follows:

$$
\begin{aligned}
x_{1}^{*} & =\frac{m_{1}^{(k-1)}\left(1-m_{2}\right)}{1-m_{2} m_{1}^{(k-1)}} \\
x_{2}^{*} & =m_{2} x_{1}^{*}+1-m_{2} \\
x_{3}^{*} & =m_{1}\left(m_{2} x_{1}^{*}+1-m_{2}\right) \\
x_{4}^{*} & =m_{1}^{2}\left(m_{2} x_{1}^{*}+1-m_{2}\right) \\
& \ldots \\
x_{k}^{*} & =m_{1}^{(k-2)}\left(m_{2} x_{1}^{*}+1-m_{2}\right)
\end{aligned}
$$

This cycle exists as long as $x_{k}^{*}$ satisfies $x_{k}^{*}>d_{2}$ in the standard conditions, and the border collision $x_{k}^{*}=d_{2}$ gives the standard closure condition. To find a simpler expression of $x_{k}^{*}$ let us proceed as follows: we apply to the discontinuity point $d_{2}$, 
in the order, the functions $T_{R}, T_{L}, T_{R}, \ldots, T_{R}$ :

$$
\begin{aligned}
p_{1} & =d_{2} \\
p_{2} & =m_{1} d_{2} \\
p_{3} & =m_{2} m_{1} d_{2}+\left(1-m_{2}\right) \\
p_{4} & =m_{1}\left(m_{2} m_{1} d_{2}+1-m_{2}\right) \\
& \ldots \\
p_{k+1} & =m_{1}^{(k-2)}\left(m_{2} m_{1} d_{2}+1-m_{2}\right)
\end{aligned}
$$

thus the standard final border collision bifurcation curve (collision of $x_{k}^{*}$ with the discontinuity point $d_{2}$ ) has the following equation:

$$
d_{2}=m_{1}^{(k-2)}\left(m_{2} m_{1} d_{2}+1-m_{2}\right)
$$

or, rearranging:

$$
m_{2}=\frac{d_{2}-m_{1}^{(k-2)}}{m_{1}^{(k-2)}\left(m_{1} d_{2}-1\right)}
$$

We also have the following expressions for the periodic points:

$$
\begin{aligned}
x_{1}^{*} & =m_{1} x_{k}^{*} \\
x_{2}^{*} & =m_{2} m_{1} x_{k}^{*}+\left(1-m_{2}\right) \\
x_{3}^{*} & =m_{1}\left(m_{2} m_{1} x_{k}^{*}+1-m_{2}\right) \\
x_{4}^{*} & =m_{1}^{2}\left(m_{2} m_{1} x_{k}^{*}+1-m_{2}\right) \\
& \ldots \\
x_{k-1}^{*} & =m_{1}^{(k-3)}\left(m_{2} m_{1} x_{k}^{*}+1-m_{2}\right) \\
x_{k}^{*} & =\frac{m_{1}^{(k-2)}\left(1-m_{2}\right)}{1-m_{2} m_{1}^{(k-1)}}
\end{aligned}
$$

so that the final border collision bifurcation curve occurring for $\delta_{R}>\delta_{R}^{*}$ and due to the collision of the periodic point $x_{k}^{*}$ with the boundary of the basin of the origin, has the following equation:

$$
\frac{m_{1}^{(k-2)}\left(1-m_{2}\right)}{1-m_{2} m_{1}^{(k-1)}}=\frac{d_{1}}{m_{1}}
$$


that is, rearranging:

$$
m_{2}=\frac{d_{1}-m_{1}^{(k-1)}}{m_{1}^{(k-1)}\left(d_{1}-1\right)}
$$

Similarly we can reason for the other principal $k$-cycles. Let us write here only the bifurcation curves, since the proof is quite similar to the one given above: they only differ in the order of application of the maps, that is we use $T_{R}, T_{L}, \ldots, T_{L}$ instead of $T_{L}, T_{R}, \ldots, T_{R}$. The starting bifurcation curve of a $k$-cycle, for $\delta_{B}<\delta_{B}^{*}$, has equation

$$
d_{2}=m_{2}^{(k-1)} m_{1} d_{2}+\left(1-m_{2}^{(k-1)}\right)
$$

or, rearranging:

$$
m_{1}=\frac{d_{2}-1+m_{2}^{(k-1)}}{d_{2} m_{2}^{(k-1)}}
$$

The standard final bifurcation curve (collision of $x_{k}^{*}$ with the discontinuity point $\left.d_{2}\right)$ is given by:

$$
d_{2}=m_{2}^{(k-2)}\left(m_{1} m_{2} d_{2}+m_{1}\left(1-m_{2}\right)\right)+\left(1-m_{2}^{(k-2)}\right)
$$

or, rearranging:

$$
m_{1}=\frac{d_{2}-1+m_{2}^{(k-2)}}{\left(m_{2} d_{2}+1-m_{2}\right) m_{2}^{(k-2)}}
$$

Instead, the border collision bifurcation curve, occurring for $\delta_{B}>\delta_{B}^{*}$ due to the collision of the $k$-cycle with the boundary of the basin of the origin, differently from the previous case, is now detected by the collision of the periodic point $x_{1}^{*}$ with the boundary $\left(d_{1}^{-1}=\frac{d_{1}}{m_{1}}\right)$, as the periodic points are now given by $x_{1}^{*}>d_{2}, x_{i}^{*}<d_{2}$ for $i=2, \ldots, k$. This border collision curve has the following equation:

$$
x_{1}^{*}=\frac{1-m_{2}^{(k-1)}}{1-m_{1} m_{2}^{(k-1)}}=\frac{d_{1}}{m_{1}}
$$

that is, rearranging:

$$
m_{1}=\frac{d_{1}}{1-m_{2}^{(k-1)}\left(1-d_{1}\right)}
$$

By using the bifurcation curves given above we have drawn all the bifurcation curves of the principal $k$-cycles for $k=2, \ldots, 15$ reported in Fig. 4 . For $k=2$ the formulas (8) and (9) give the same bifurcation curves of the formulas in (11) and (12), which is obvious as the 2 -cycle is the unique one with one point on the right 

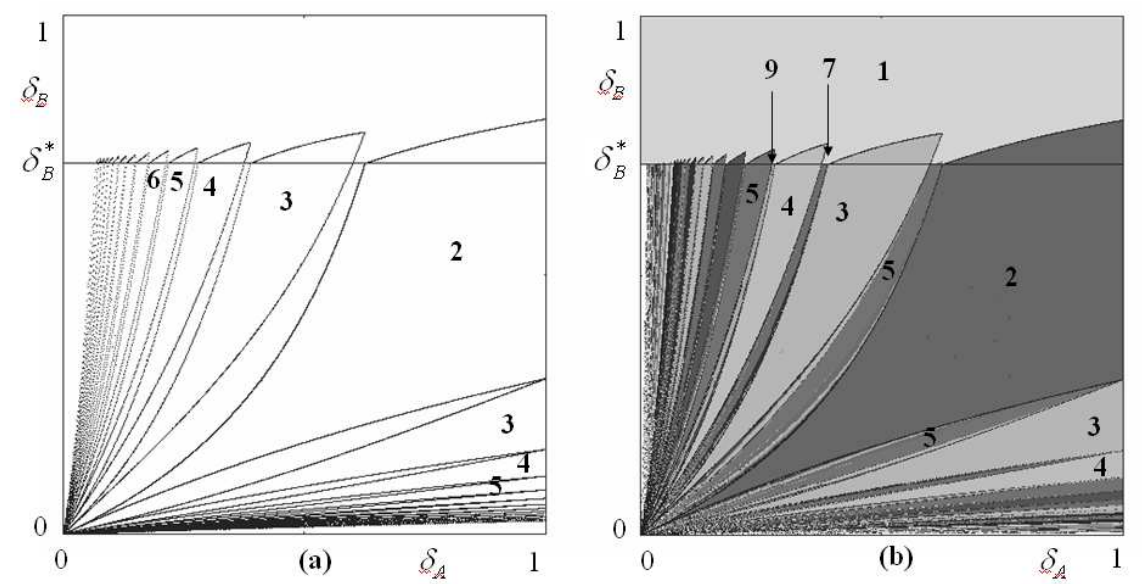

Figure 4. Map ((a) Bifurcation curves of first degree in the parameter plane $\left(\delta_{A}, \delta_{B}\right)$, for $d_{1}=0.2$ and $d_{2}=0.7$, for cycles of periods $2, \ldots, 15$, obtained by the analytical expressions calculated for the map $T$. (b) The tongues of periodicity, bounded by the same bifurcation curves as in (a), are numerically obtained with gray tonalities associated with the different periods.

and one on the left of the discontinuity $d_{2}$, and can be detected by labelling the periodic points as $x_{1}^{*}<d_{2}$ and $x_{2}^{*}>d_{2}$ in the first case, or labelling as $x_{1}^{*}>d_{2}$ and $x_{2}^{*}<d_{2}$ as in the second case. So, what is called initial and final in the first case, can be called final and initial, respectively, in the second one. The border collision of the cycle with the boundary of the basin of attraction of the origin may thus involve both the periodic points of the 2 -cycle: it is given by the collision $x_{2}^{*}=\frac{d_{1}}{m_{1}}$ in the first case (from the equation in (10)) and the collision $x_{1}^{*}=\frac{d_{1}}{m_{1}}$ in the second case (from the equation (13)).

Instead, for $k=3, \ldots, 15$, all the bifurcation curves in Fig. 4 located above the tongue of the 2-cycle can be drawn by the formulas in (8), (9) and in (10), and those below the period-2 tongue can be drawn by (11), (12) and (13). Notice that the formulas given in (8), (9) and in (10), as well as those in (11), (12) and (13), hold whichever are the positions of the discontinuity points $d_{1}$ and $d_{2}$. In Fig. 5 we show the bifurcation curves for $k=2, \ldots, 15$ in a different case. It is worth noticing that reasoning in the same way it is possible to find also the boundaries of the other 

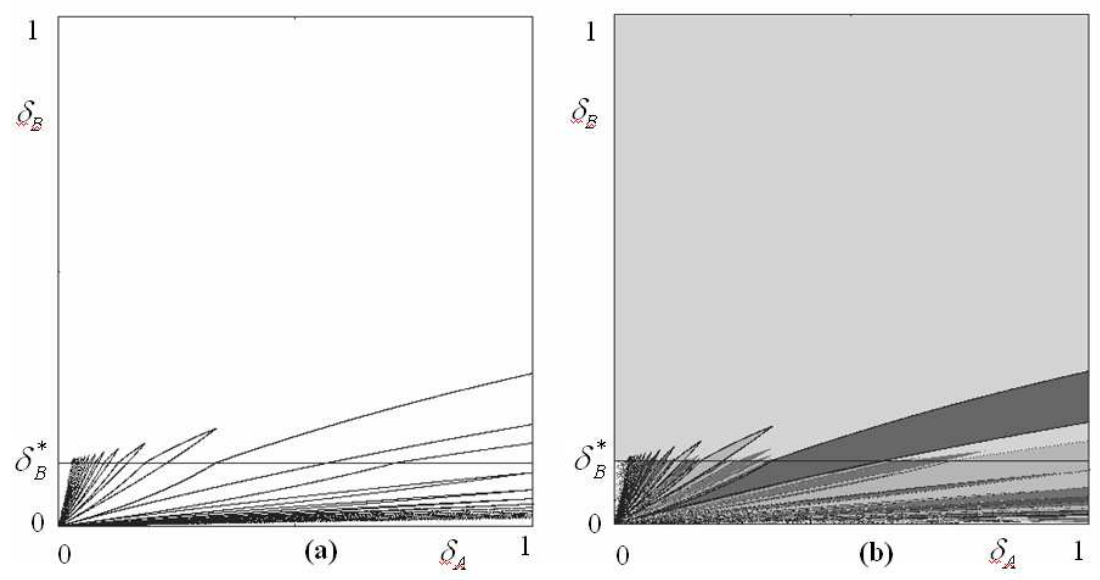

Figure 5. (a) Bifurcation curves of first degree of complexity for cycles of periods $2, \ldots, 15$, in the parameter plane $\left(\delta_{A}, \delta_{B}\right)$, obtained by the analytical expressions for the map $T$ with $d_{1}=0.7$ and $d_{2}=0.7$. (b) The tongues of periodicity, bounded by the same bifurcation curves as in (a), are numerically obtained with gray tonalities associated with the different periods.

bifurcation curves. In fact, besides the regions associated with the "tongues of first degree" there are infinitely many other periodicity tongues.

Indeed, between any two tongues having periods $k_{1}$ and $k_{2}$ there exists a tongue having period $k_{1}+k_{2}$ (see the numbers in Fig. 4). Moreover, all the periodicity tongues are disjoint, i.e. no overlapping occurs, which means that we can at most have only one $k$-cycle, with $k>1$, for each fixed set of parameters.

To be more specific, in the description of the periodicity tongues we can associate a number to each region, which may be called "rotation number", in order to classify all the periodicity tongues. In this notation a periodic orbit of period $k$ is characterized not only by the period but also by the number of points in the two branches separated by the discontinuity point (denoted by $T_{L}$ and $T_{R}$ respectively). So, we can say that a cycle has a rotation number $\frac{p}{k}$ if a $k$-cycle has $p$ points on the $L$ side and the other $(k-p)$ on the $R$ side. Then between any pair of periodicity 
regions associated with the "rotation number" $\frac{p_{1}}{k_{1}}$ and $\frac{p_{2}}{k_{2}}$ there exists also the periodicity tongue associated with the "rotation number" $\frac{p_{1}}{k_{1}} \oplus \frac{p_{2}}{k_{2}}=\frac{p_{1}+p_{2}}{k_{1}+k_{2}}$ (also called Farey composition rule $\oplus$, see for example in [21]).

Then, following Leonov ([26], [27]) (see also Mira [31], [32]), between any pair of contiguous "tongues of first degree", say $\frac{1}{k_{1}}$ and $\frac{1}{k_{1}+1}$, we can construct two infinite families of periodicity tongues, called "tongues of second degree" by the sequence obtained by adding with the Farey composition rule $\oplus$ iteratively the first one or the second one, i.e. $\frac{1}{k_{1}} \oplus \frac{1}{k_{1}+1}=\frac{2}{2 k_{1}+1}, \frac{2}{2 k_{1}+1} \oplus \frac{1}{k_{1}}=\frac{3}{3 k_{1}+1}, \ldots$ and so on, that is:

$$
\frac{n}{n k_{1}+1} \text { for any } n>1
$$

and $\frac{1}{k_{1}} \oplus \frac{1}{k_{1}+1}=\frac{2}{2 k_{1}+1}, \frac{2}{2 k_{1}+1} \oplus \frac{1}{k_{1}+1}=\frac{3}{3 k_{1}+2}, \frac{3}{3 k_{1}+2} \oplus \frac{1}{k_{1}+1}=\frac{4}{4 k_{1}+3} \ldots$, that is:

$$
\frac{n}{n k_{1}+n-1} \text { for any } n>1
$$

which give two sequences of tongues accumulating on the boundary of the two starting ones.

Clearly, this mechanisms can be repeated: between any pair of contiguous " tongues of second degree", for example $\frac{n}{n k_{1}+1}$ and $\frac{n+1}{(n+1) k_{1}+1}$, we can construct two infinite families of periodicity tongues, called "tongues of third degree" by the sequence obtained by adding with the composition rule $\oplus$ iteratively the first one or the second one. And so on. All the rational numbers are obtained in this way, giving all the infinitely many periodicity tongues.

Besides the notation used above, called method of the rotation numbers, we may also follow a different approach, related with the symbolic sequence associated to a cycle. In this notation, considering the principal tongue of a periodic orbit of period $k$ constituted by one point on the $L$ side and $(k-1)$ on the $R$ side, we associate to the cycle the symbol sequence LR..(k-1 times).R. Then the composition of two consecutive cycles is given by:

$$
L R . .(k-1 \text { times }) . R \oplus L R . .(k \text { times }) . R=L R . .(k-1 \text { times }) . R L R . .(k \text { times }) . R
$$

that is, the two sequences are just put together in file (and indeed this sequence of bifurcations is also called "boxes in files" in [32]), and the sequence of maps to apply in order to get the cycle are listed from left to right. More generally, it is true that given a periodicity tongue associated with a symbols' sequence $\sigma$ (consisting of letters $L$ and $R$, giving the cycle from left to right) and a second one with a symbols' 
sequence $\tau$, then also the composition of the two sequences exists, associated with a periodicity tongue with symbols' sequence $\sigma \tau$ :

$$
\sigma \oplus \tau=\sigma \tau
$$

It is worth to notice that these periodicity tongues never overlap, and this implies that coexistence of different periodic cycles is not possible.

\section{The Discontinuous maP AS LIMITING CASE OF A FAMILY OF CONTINUOUS} MAPS

The map so far considered can be seen as the limiting case of the continuous map (1) as $\lambda \rightarrow+\infty$ when the payoff functions $A, B:[0,1] \rightarrow \mathbb{R}$ are such that

$$
\begin{array}{lll}
A(x)>B(x) & \text { if } & d_{1}<x<d_{2} \\
A(x)=B(x) & \text { if } & x=d_{1} \text { or } x=d_{2} \\
A(x)<B(x) & \text { if } & x<d_{1} \text { or } x>d_{2}
\end{array}
$$

In this section we are interested to analyze the behavior of the continuous map (1) for increasing values of $\lambda$ up to the limiting case $\lambda \rightarrow+\infty$.

For our analysis it is sufficient to notice that if both functions $A$ and $B$ are continuous, then also the map $f$ is continuous in the whole interval $[0,1]$, and its graph is contained in the strip bounded by two lines, being $\left(1-\delta_{B}\right) x \leq f(x) \leq$ $\left(1-\delta_{A}\right) x+\delta_{A}$. However, even if $B(x)$ and $A(x)$ are smooth functions, the map $f$ in general is not smooth in the considered interval, since $f$ is not differentiable where the functions $A$ and $B$ intersect.

In order to obtain some insight into the effect of increasing values of $\lambda$, we consider the map (1) with $g(z)=\frac{2}{\pi} \arctan (\lambda z)$, as in $([6])$, and then we gradually move to the limiting case $\lambda \rightarrow+\infty$, equivalent to consider $g(\cdot)=1$, so that the dynamical system (1) assumes the form (3). So, beyond its intrinsic interest, the map (3) we have studied in this paper provides several insights into the asymptotic properties of the continuous map for high values of $\lambda$ and emphasizes the role the parameters $\delta_{A}$ and $\delta_{B}$ play in the dynamics. For example, under some conditions on function $A$ and $B$, increasing values of $\lambda$ cause the loss of stability of the equilibrium via a a flip bifurcation that opens the usual route to chaos through a period doubling cascade. However, as shown in [7], such a chaotic behavior can only be observed for intermediate values of the parameter $\lambda$, as the asymptotic dynamics settles on 
a stable periodic cycle for sufficiently high values of $\lambda$. This can be numerically observed for many different values of the parameters $\delta_{A}$ and $\delta_{B}$, the only difference being the period of the stable cycle that prevails at high values of $\lambda$. Fig. 6 illustrates how the continuous map (1) changes as $\lambda$ varies. It is also interesting to observe how

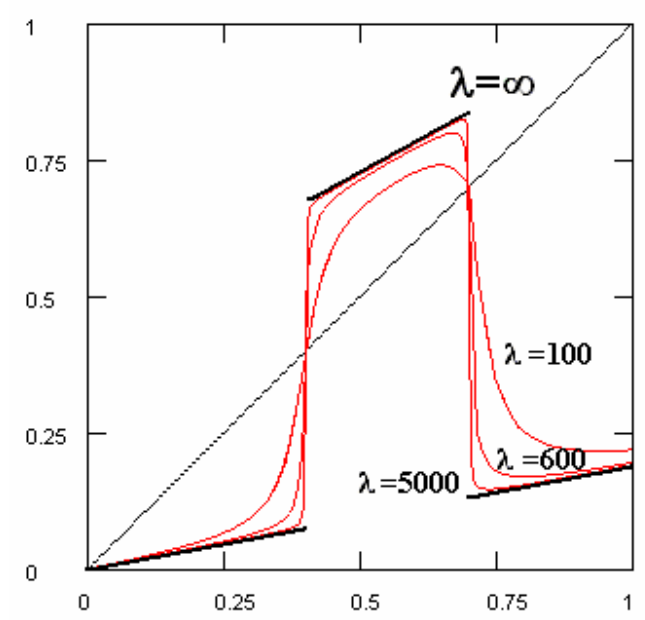

Figure 6. The map $f$ defined in (1) with $g(z)=\frac{2}{\pi} \arctan (\lambda z)$ and for three different values of parameter $\lambda$, compared with the limiting map obtained for $\lambda=\infty$.

the corresponding two-dimensional bifurcation diagram in the parameters' plane $\left(\delta_{A}, \delta_{B}\right)$ evolves as $\lambda$ increases. The different gray tonalities shown in the three pictures of Fig. 7, obtained with $\lambda=100, \lambda=600, \lambda=5000$ respectively, represent the kind of asymptotic behaviour numerically observed: convergence to the stable fixed point or a stable periodic cycle of low period when the parameters are chosen in the white or gray regions (with different tonalities representing different periods) or the convergence to a chaotic attractor, or periodic cycle of very high period, when the parameters are chosen in the white regions. It can be seen that chaotic behavior becomes more common at intermediate values of $\lambda$ whereas periodic cycles of low period prevail for very high values of $\lambda$. 

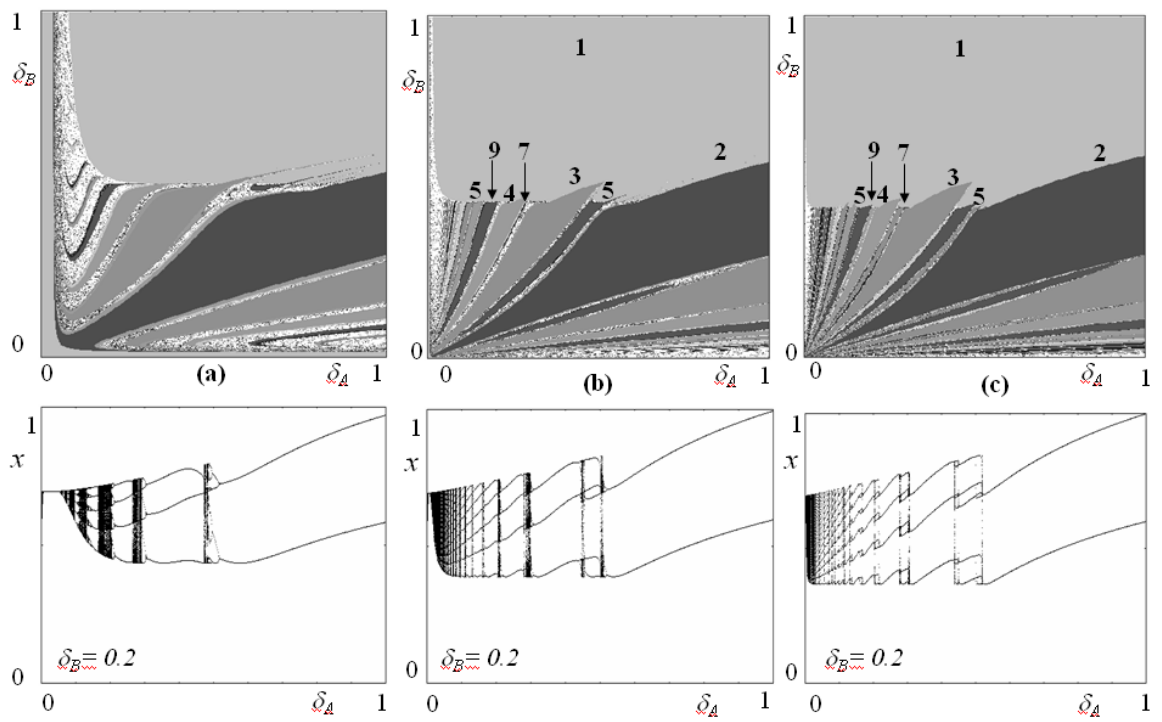

Figure 7. Bifurcation diagrams in the parameters' plane $\left(\delta_{A}, \delta_{B}\right)$ for the continuous map $g(z)=\frac{2}{\pi} \arctan (\lambda z)$ with (a) $\lambda=100$ (b) $\lambda=600$ (c) $\lambda=5000$. The grey shaded regions represent periodic tongues of low periods, whereas in the white regions the asymptotic behavior is periodic with high periods or aperiodic, i.e. chaotic, as shown in the corresponding one-dimensional bifurcation diagrams shown in the lower part of the figure, obtained with increasing values of $\delta_{A}$ and a fixed value $\delta_{B}=0.2$.

\section{Conclusions}

The main goal of this paper was the analytic description of the bifurcations occurring in a piecewise linear map $T:[0,1] \rightarrow[0,1]$ formed by three portions with two different slopes, $m_{i} \in[0,1], i=1,2$, separated by two discontinuity points $0 \leq d_{1}<d_{2} \leq 1$. Firstly we recalled how this piecewise continuous map which depends on four parameters can be interpreted as the generalization of a simpler map with one discontinuity. In fact, we showed how both the bifurcation diagram and the analytic expression of the periodicity tongues of first degree maintain some important aspects of the map with only one discontinuity point. Secondly, we examined the different dynamic behaviors of the map and gave a characterization 
of the structure of the basins. In particular, by using methods already known in the literature, we have then studied the effects, on the structure of the border collision bifurcation curves, induced by the introduction of the second discontinuity point. Finally we showed how the map we examined can be seen as the limit case of a continuos map, and showed how close the bifurcation curves of the limiting case are similar to those of the continuous model, with a high value of the parameter $\lambda$.

The methods followed to obtain the analytic expressions are quite general and can be easily generalized to cases with different linear functions and several discontinuities, and with slopes different from the ones considered in the model studied in this paper.

\section{REFERENCES}

[1] V. Avrutin and M. Schanz, Multi-parametric bifurcations in a scalar piecewise-linear map, Nonlinearity, 19 (2006), 531-552.

[2] V. Avrutin, M. Schanz and S. Banerjee, Multi-parametric bifurcations in a piecewise-linear discontinuous map, Nonlinearity, 19 (2006), 1875-1906.

[3] S. Banerjee and C. Grebogi, Border-collision bifurcations in two-dimensional piecewise smooth maps, Physical Review E, 59(4) (1999), 4052-4061.

[4] S. Banerjee, M.S. Karthik, G. Yuan and J.A. Yorke, Bifurcations in One-Dimensional Piecewise Smooth Maps - Theory and Applications in Switching Circuits, IEEE Trans. Circuits Syst.-I: Fund. Theory Appl. 47(3) (2000), 389-394.

[5] S. Banerjee, P. Ranjan, and C. Grebogi, Bifurcations in 2D Piecewise Smooth Maps - Theory and Applications in Switching Circuits, IEEE Trans. Circuits Syst.-I: Fund. Theory Appl. $\mathbf{4 7}(5)$ (2000), 633-64.

[6] G.I. Bischi and U. Merlone, Global dynamics in binary choice models with social influence, Journal of Mathematical Sociology (in press).

[7] G.I. Bischi, L. Gardini and U. Merlone, Impulsivity in binary choices and the emergence of periodicity, Discrete Dynamics in Nature and Society (in press).

[8] P. Collet. and J.P. Eckmann, Iterated maps on the interval as dynamical systems, Birkhauser, Boston (1980).

[9] R. Day, Irregular growth cycles, The American Economic Review 72(3) (1982), 406-414.

[10] R. Day, "Complex Economic Dynamics", MIT Press, Cambridge, 1994.

[11] R.L. Devaney, "An Introduction to Chaotic Dynamical Systems", The Benjamin/Cummings Publishing Co., Menlo Park, California (1986).

[12] M. di Bernardo, M.I. Feigen, S.J. Hogan and M.E. Homer, Local analysis of C-bifurcations in n-dimensional piecewise smooth dynamical systems, Chaos, Solitons \& Fractals 10(11) (1999), 1881-1908. 
PERIODIC CYCLES AND BIFURCATION .. — JDSGT VOL. 7, NUMBER 2 (2009) 12

[13] M. di Bernardo, C.J. Budd, A.R. Champneys and P. Kowalczyk, "Piecewise-smooth Dynamical Systems Theory and Applications", Springer-Verlag, London, 2008.

[14] O. Feely, D. Fournier-Prunaret, I. Taralova-Roux and D. Fitzgerald, Nonlinear dynamics of bandpass sigma-delta modulation. An investigation by means of the critical lines tool, International Journal of Bifurcation and Chaos, 10(2) (2000), 303-327.

[15] D. Fournier-Prunaret, O.Feely, I. Taralova-Roux, Lowpass sigma-delta modulation : an analysis by means of the critical lines tool, Non Linear Analysis, 47 (2001), 5343-5355.

[16] M. Gallegati, L. Gardini, L., T. Puu, and I. Sushko, Hicks's trade cycle revisited: cycles and bifurcations, Mathematics and Computers in Simulations, 63 (2003), 505-527.

[17] L. Gardini, I. Sushko and A. Naimzada, Growing Through Chaotic Intervals, Journal of Economic Theory, 143 (2008), 541-557.

[18] Granovetter M., (1978), "Threshold Models of Collective Behavior", The American Journal of Sociology 83(6), 1420-1443.

[19] J. Guckenheimer. and P. Holmes, "Nonlinear Oscillations, Dynamical Systems, and Bifurcations of Vector Fields", Springer-Verlag, (1983).

[20] C. Halse, M. Homer and M. di Bernardo, C-bifurcations and period-adding in one-dimensional piecewise-smooth maps, Chaos, Solitons \& Fractals 18 (2003), 953-976.

[21] B.L. Hao, "Elementary Symbolic Dynamics and Chaos in Dissipative Systems", World Scientific, Singapore, 1989.

[22] C.H. Hommes, A reconsideration of Hick's non-linear trade cycle model, Structural Change and Economic Dynamics 6 (1995), 435-459.

[23] C.H. Hommes and H.E. Nusse, Period three to period two bifurcations for piecewise linear models, Journal of Economics 54(2) (1991), 157-169.

[24] C.H. Hommes, H.E. Nusse and A. Simonovits, Cycles and chaos in a socialist economy, Journal of Economic Dynamic and Control, 19 (1995), 155-179.

[25] P. Jain and S. Banerjee, Border-collision bifurcations in one-dimensional discontinuous maps, Int. Journal of Bifurcation and Chaos, 13(11) (2003), 3341-3351.

[26] N.N. Leonov, Map of the line onto itself, Radiofisica 3(3) (1959), 942-956.

[27] N.N. Leonov, Discontinuous map of the straight line, Dohk. Ahad. Nauk. SSSR. 143(5) (1962), 1038-1041.

[28] Y.L. Maistrenko, V.L. Maistrenko and L.O. Chua, Cycles of chaotic intervals in a timedelayed Chua's circuit, International Journal of Bifurcation and Chaos 3(6) (1993), 15571572 .

[29] Y.L. Maistrenko, V.L. Maistrenko, S.I. Vikul and L. Chua, Bifurcations of attracting cycles from time-delayed Chua's circuit, International Journal of Bifurcation and Chaos 5(3) (1995), 653-671.

[30] Y.L. Maistrenko, V.L. Maistrenko and S.I. Vikul, On period-adding sequences of attracting cycles in piecewise linear maps, Chaos, Solitons \& Fractals 9(1) (1998), 67-75.

[31] C. Mira, Sur les structure des bifurcations des diffeomorphisme du cercle, C.R.Acad. Sc. Paris, 287, Series A ( 1978), 883-886. 
[32] C. Mira, "Chaotic Dynamics", World Scientific, Singapore, 1987.

[33] H.E. Nusse and J.A. Yorke, Border-collision bifurcations including period two to period three for piecewise smooth systems, Physica D 57 (1992), 39-57.

[34] H.E. Nusse and J.A. Yorke, Border-collision bifurcations for piecewise smooth onedimensional maps, International Journal of Bifurcation and Chaos 5(1) (1995), 189-207.

[35] T. Puu and I. Sushko Ed.s, "Oligopoly and Complex Dynamics: Models and Tools", SpringerVerlag, New York, 2002.

[36] T. Puu and I. Sushko Ed.s, "Business Cycles Dynamics: Models and Tools", Springer-Verlag, New York, 2006.

[37] T. Puu, The Hicksian trade cycle with floor and ceiling dependent on capital stock, Journal of Economic Dynamics \& Control, 31 (2007), 575-592.

[38] T. Puu, L. Gardini and I. Sushko, A Hicksian multiplier-accelerator model with floor determined by capital stock, Journal of Economic Behavior and Organization 56 (2005), 331-348.

[39] C. Robinson, "Dynamical Systems", CRC Press (1995).

[40] T.C. Schelling, Hockey helmets, concealed weapons and daylight saving, Journal of Conflict Resolution 17(3) (1973), 381-428.

[41] T.C. Schelling, "Micromotives and Macrobehavior", W.W. Norton, New York, 1978.

[42] I. Sushko, A. Agliari and L. Gardini, Bistability and border-collision bifurcations for a family of unimodal piecewise smooth maps, Discrete and Continuous Dynamical Systems, Serie B, $\mathbf{5}(3)$ (2005), 881-897.

[43] I. Sushko, A. Agliari and L. Gardini, Bifurcation Structure of Parameter Plane for a Family of Unimodal Piecewise Smooth Maps: Border-Collision Bifurcation Curves, Chaos, Solitons \& Fractals, 29(3), (2006), 756-770.

[44] I. Sushko, L. Gardini and T. Puu, Tongues of Periodicity in a Family of Two-dimensional Discontinuous Maps of Real Möbius Type, Chaos, Solitons \& Fractals 21 (2004), 403-412.

[45] I. Sushko, T. Puu and L. Gardini, The Hicksian floor-roof model for two regions linked by interregional trade, Chaos Solitons \& Fractals, 18 (2003), 593-612.

[46] I. Sushko, L. Gardini and T. Puu, Regular and Chaotic Grouwth Cyckles in a Hicksian Floor/Roof Model, Journal of Economic Behavior and Organization, (2009) (to appear).

[47] F. Tramontana, L. Gardini and T. Puu, Duopoly games with alternative technologies, Journal of Economic Dynamic and Control 33 (2008), 250-265.

[48] F. Tramontana, L. Gardini, G.I. Bischi Bifurcation curves in discontinuous maps, Discrete and Continuous Dynamical Systems (in press).

[49] S. Wiggins, "Introduction to applied nonlinear dynamical systems and chaos", SpringerVerlag, N.Y. (1990).

[50] Z.T. Zhusubaliyev and E. Mosekilde, "Bifurcations and Chaos in Piecewise - Smooth Dynamical Systems", World Scientific, Singapore, 2003.

[51] Z.T. Zhusubaliyev, E. Mosekilde, S. Maity, S. Mohanan and S. Banerjee, Border collision route to quasiperiodicity: Numerical investigation and experimental confirmation, Chaos 16, 023122 (2006), 1-11. 
PERIODIC CYCLES AND BIFURCATION ... - JDSGT VOL. 7, NUMBER 2 (2009) 123

[52] Z.T. Zhusubaliyev, E. Soukhoterin and E. Mosekilde, Quasiperiodicity and torus breakdown in a power electronic dc/dc converter, Mathematics and Computers in Simulation 73 (2007) 364-377. 